\title{
Expression of HLA-E molecules in the placental tissue of women infected with HIV-1 and uninfected women
}

\author{
Juliana Martinez a , Mariana Rodrigues Santiago a, Gustavo Martelli-Palomino b, \\ Diego Agra de Souza ${ }^{\mathrm{c}}$, Társia Giabardo Alves Silva ${ }^{\mathrm{d}}$, Gyl Eanes Barros Silva ${ }^{\mathrm{c}}$, \\ Fernando Chahud ${ }^{\mathrm{c}}$, Eduardo Antônio Donadi ${ }^{\mathrm{e}}$, Ana Paula Morais Fernandes ${ }^{\mathrm{a},}$ * \\ ${ }^{a}$ University of São Paulo at Ribeirão Preto College of Nursing, General and Specialized Nursing Department, Avenida dos Bandeirantes, 3900, Monte Alegre, \\ Ribeirão Preto, CEP 14040-902, SP, Brazil \\ b Departamento de Análises Clínicas e Toxicológicas, Universidade Federal do Rio Grande do Norte (UFRN), Natal, Brazil \\ ${ }^{\mathrm{c}}$ University of São Paulo at Ribeirão Preto School of Medicine, Pathology Department, Avenida dos Bandeirantes, 3900, Monte Alegre, Ribeirão Preto, SP, \\ Brazil \\ d School of Pharmaceutical Sciences, Universidade Estadual Paulista Júlio de Mesquita Filho, Rodovia Araraquara, Jaú Km 1, Campos Ville, Araraquara, SP, \\ Brazil \\ e University of São Paulo at Ribeirão Preto School of Medicine, Medical Clinical Department, Avenida dos Bandeirantes, 3900, Monte Alegre, Ribeirão Preto, \\ SP, Brazil
}

\section{A R T I C L E I N F O}

\section{Article history:}

Received 24 November 2015

Received in revised form

25 July 2016

Accepted 22 August 2016

\section{Keywords:}

HLA-E

Expression

Placenta

HIV-1

\begin{abstract}
A B S T R A C T
Introduction: Expression of HLA-E molecule in the placental extravillous trophoblast is associated with immune system cell inhibition, resulting in immune tolerance to fetus during pregnancy. HIV- 1 can infect trophoblast cells and modify the expression of HLA-E, which may inhibit the cytotoxic activity of the immune system.

Aim: The aim of this study was to evaluate HLA-E expression in third trimester placental tissue of women infected with HIV-1 and uninfected women.

Methods: We performed an immunohistochemistry assay to evaluate HLA-E staining in the placental tissue of 99 HIV-1 infected and 85 uninfected women. A pathologist analyzed and classified the HLA-E expression in the placental cells.

Results: Irrespective of the HIV status, HLA-E staining was observed in the extravillous trophoblast cells, endothelial cells and Hofbauer cells, but not in the syncytiotrophoblast. HLA-E staining showed no significant difference between the placental tissue of women infected with HIV-1 and uninfected women $(P=0.76)$. Considering HIV-1 infected women, HLA-E staining was not influenced by HIV-1 viral load $(P=0.48), \mathrm{CD}^{+} \mathrm{T}$-cell count $(P=0.10)$ and antiretroviral therapy used during pregnancy $(P=0.54)$. Discussion: Despite the presence of HIV-1 infection, the expression of HLA-E molecules in the placental tissue was not modified when the infection was under antiretroviral therapy control.
\end{abstract}

() 2016 Published by Elsevier Ltd.

\section{Introduction}

The human leukocyte antigen E (HLA-E) is a nonclassical class I molecule encoded by a gene of the major histocompatibility complex (MHC). The molecule may modulate the immune response in several pathological conditions, including tumors and viral infections, and in physiological conditions such as pregnancy [1,2]. The expression of nonclassical HLA class I molecules in the

\footnotetext{
* Corresponding author.

E-mail address: anapaula@eerp.usp.br (A.P.M. Fernandes).
}

extravillous trophoblast (EVT) cells of the placenta contributes to maternal tolerance against paternal antigens, permitting a successful pregnancy [3-5]. In pregnant women infected with human immunodeficiency virus-1 (HIV-1), studies indicate that the virus can also infect the EVT cells [6], and the presence of the HIV-1 p24 $14-22$ peptide can increase the expression of HLA-E in lymphocytes $[7,8]$, suggesting that the virus may control the expression of the molecule. The interaction of HLA-E with the natural killer (NK) receptors may inhibit NK cell cytotoxic activity, representing an immune response evasion strategy of HIV-1 [7,8].

Considering that HIV-1 can infect EVT cells and that the viral 
infection can modulate HLA-E expression, it is relevant to analyze whether the EVT cells of women infected with HIV-1 exhibit altered expression of HLA-E when compared to those of uninfected women. Therefore, this study aimed to evaluate the expression of HLA-E in the placental tissue of women infected with HIV-1 and uninfected women.

\section{Methods}

\subsection{Ethical aspects}

The study was approved by the Ethics Committee of the College of Nursing of Ribeirão Preto, University of São Paulo, Brazil (protocol \# 1330/2011), and all participants signed the Free and Informed Consent Form. This study was carried out in accordance with The Code of Ethics of the World Medical Association (Declaration of Helsinki) for experiments involving humans.

\subsection{Population samples}

We analyzed 184 fragments of paraffin-embedded placental tissue, of which 99 fragments were from HIV-1-infected women $(\mathrm{HIV}+)$ and 85 from uninfected women (HIV-). All placentas were obtained from successful third trimester (37 weeks or older) pregnancies.

\subsection{Clinical data}

Clinical and laboratory data of the HIV + group, such as viral load, circulating $\mathrm{CD} 4^{+} \mathrm{T}$ lymphocyte count ( $\mathrm{CD} 4^{+} \mathrm{T}$-cells), and type of antiretroviral therapy (ART) used during pregnancy were retrieved from patients' medical records available at the electronic files of the University Hospital of the Faculty of Medicine of Ribeirão Preto, University of São Paulo, Brazil.

\subsection{Immunohistochemistry assay}

Fragments of paraffin-embedded placental tissue were cut into $3-\mu \mathrm{m}$ sections, fixed on silanized slides, and subjected to immunohistochemical assay to evaluate the expression of HLA-E molecules.

Tissue fragments were incubated at $60^{\circ} \mathrm{C}$ for $1 \mathrm{~h}$ and subjected to xylol and alcohol washing for complete deparaffinization and hydration. For antigen recovery, citrate solution ( $\mathrm{pH}$ 6.0) was used for $40 \mathrm{~min}$ at $96{ }^{\circ} \mathrm{C}$. Endogenous peroxidase blocking was performed using phosphate-buffered saline (PBS), methanol, and 30\% hydrogen peroxide for $10 \mathrm{~min}$, and to avoid nonspecific binding, the specimens were incubated with $1 \%$ bovine serum albumin (BSA, Sigma-Aldrich, St. Louis, MO) for $5 \mathrm{~min}$. The anti-HLA-E antibody (clone MEM-E/02, EXBIO Antibodies, Prague, Czech Republic), diluted at 1:250 in a solution containing $1 \%$ BSA, was added to the specimens and incubated overnight at room temperature in a moist chamber. The specimens were then incubated with EasyLink One (EasyPath, Indaiatuba, Brazil) polymer for 30 min in dark. The specimens were then stained with diaminobenzidine (DAB) for $5 \mathrm{~min}$ and counterstained with Mayer's hematoxylin for $3 \mathrm{~min}$. Dehydration was performed using different concentrations of alcohol and xylol, and Canada balsam was used for slide mounting.

A pathologist blinded to patient identification and clinical and laboratory data analyzed the tissues and classified the magnitude of HLA-E expression in trophoblast cells by adopting the score used by Li et al. [9]. When less than 5\% of the cells expressed HLA-E molecules, the expression was considered to be negative. Positive labeling was classified as $1+(6-25 \%$ of the cells expressing HLA-E), $2+(26-50 \%), 3+(51-75 \%)$, and $4+(>75 \%)$.

\subsection{Statistical analysis}

For data analysis, chi-square test was applied with significance set at $5 \%$ and a logistic model was applied. For the analysis, the Odds Ratio (OR) was estimated with a 95\% confidence interval (95\% CI) by using the software SAS $^{\mathbb{R}} 9[10]$.

\section{Results}

HIV-1-infected women were aged $18-41$ years (mean $=28$; $\mathrm{SD} \pm 6.25$ ), and most of them were single, exhibited Caucasian ancestry, were unemployed, and had up to 9 years of schooling. Uninfected women were aged $18-38$ years ( mean $=24, \mathrm{SD} \pm 5.54$ ), and most of them presented Caucasian ancestry, had 9-12 years of schooling, were unemployed, and were married (see Table 1).

Regarding obstetric history, HIV-1-infected women gave birth to an average of 3 children (range $1-10$ ) and $20 \%$ of them had previous miscarriages, $87 \%$ had access to prenatal care, and $51 \%$ had cesarean deliveries. Uninfected women had an average of 2 children (range $1-7$ ) and $14 \%$ had miscarriages, $100 \%$ had access to prenatal care, and $74 \%$ had vaginal delivery.

Clinical and laboratory data indicated that $83.7 \%$ of HIV-1infected women had a viral load of $<10,000$ copies $/ \mathrm{mL}, 44.1 \%$ had $\mathrm{CD}^{+}{ }^{+}$-cell count between 200 and 499 cells $/ \mathrm{mm}^{3}$, and $95.1 \%$ of the patients used protease inhibitors (PI) in combination with antiretroviral drugs.

Irrespective of the HIV status, HLA-E was expressed in the human placental tissue mainly in the EVT cells, endothelial cells, and Hofbauer cells, but was not expressed in the syncytiotrophoblast (see Fig. 1).

The stratification of patients according to the HIV status revealed that the HIV + group presented a relatively higher frequency of $3+$ staining and a lower frequency of $1+$ staining, whereas the HIV- group showed a relatively higher frequency of $4+$ staining and a lower frequency of negative staining. Despite this variation, HLA-E staining of the placentas showed no significant difference between the HIV+ and HIV-groups $(P=0.76$; Table 2$)$.

The stratification of HLA-E staining in the placentas of the HIV + group according to viral load, CD4 ${ }^{+}$T-cell count, and type of ART used exhibited no significant difference (Table 2). It should be emphasized that the use of ART was analyzed according to the use or no use of PI in combination with antiretroviral drugs.

Logistic regression results also indicated no statistically

Table 1

Participant distributions in this study according to demographic parameters.

\begin{tabular}{lll}
\hline Demographic parameters & HIV $+(\%)$ & HIV- (\%) \\
\hline Marital status & 58 & 14 \\
Single & 15 & 24 \\
Married & 19 & 59 \\
Living common law & 3 & 0 \\
Separated & 4 & 3 \\
Divorced & 1 & 0 \\
Widowed & & \\
Ancestry & 68 & 53 \\
Caucasian & 20 & 47 \\
Admixture & 12 & 0 \\
African & & \\
Years of schooling & 60 & 37 \\
Up to 9 years & 30 & 63 \\
From 9 to 12 years & 6 & 0 \\
More than 12 years & 4 & 0 \\
Undeclared & & \\
Employment & 33 & 31 \\
Yes & 67 & 69 \\
No & & \\
\hline
\end{tabular}




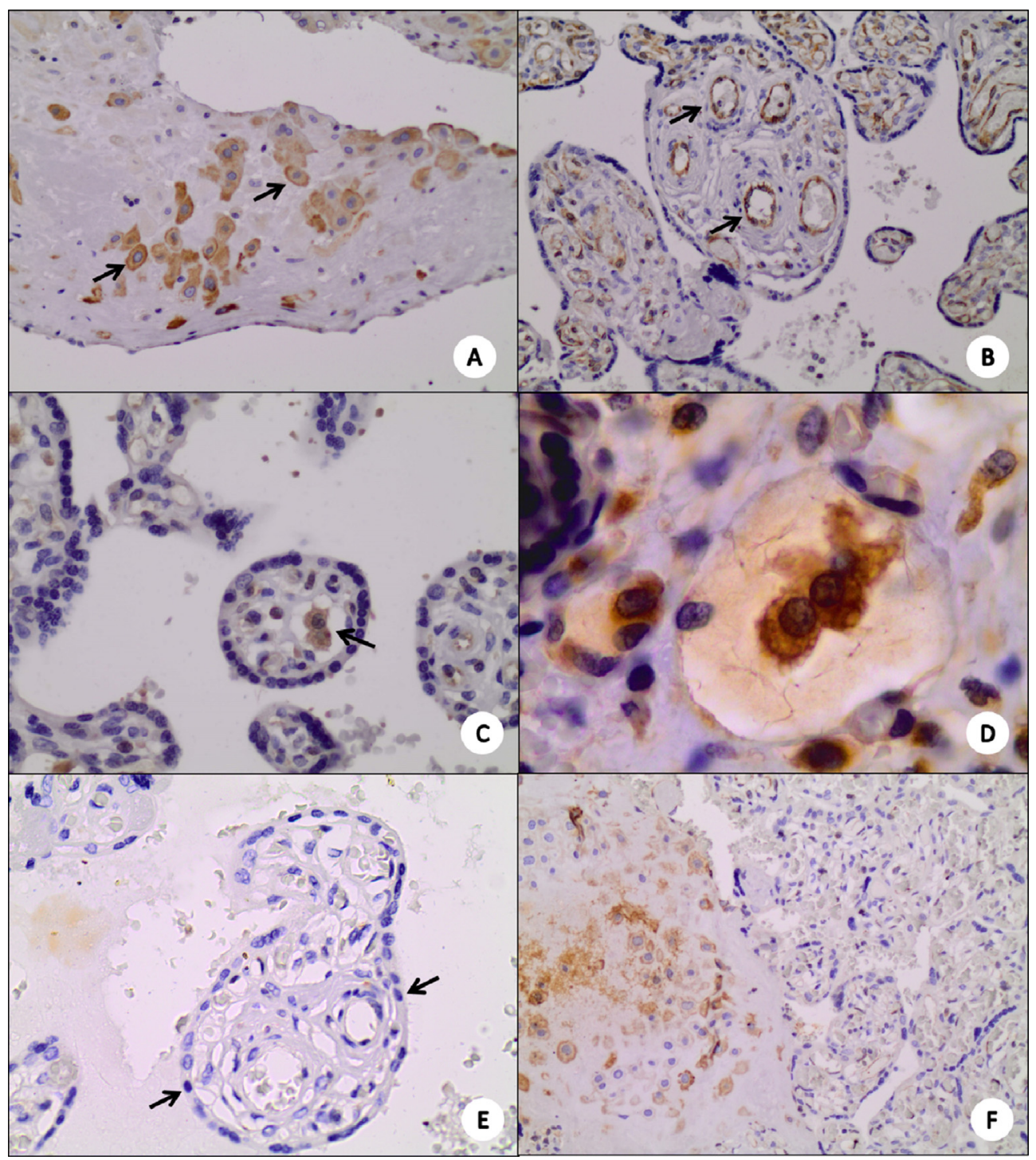

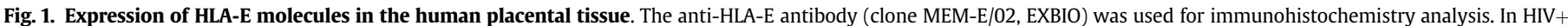

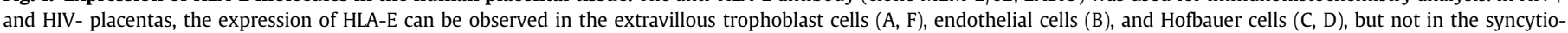
trophoblast (E, F).

Table 2

HLA-E staining frequency $(0-4+)$ stratified according to the following variables: HIV-1 status, HIV-1 viral load, CD4 ${ }^{+}$T-cell count, and type of antiretroviral regimen used by the patients.

\begin{tabular}{lllll}
\hline & \multicolumn{2}{l}{ HLA-E expression } & \multirow{2}{*}{ P-value } \\
\cline { 2 - 3 } & $0(\leq 5 \%)$ & $1,2,3(6-75 \%)$ & $4(>75 \%)$ & \\
\hline HIV-1 Status & & & & \\
Uninfected & $14.1 \%(12)$ & $60.0 \%(51)$ & $25.9 \%(22)$ & 0.76 \\
Infected & $15.2 \%(15)$ & $63.6 \%(63)$ & $21.2 \%(21)$ & \\
Viral Load & & & & \\
$<10,000$ copies/mL & $17.3 \%(14)$ & $61.7 \%(50)$ & $21.0 \%(17)$ & 0.48 \\
$>10,000$ copies $/ \mathrm{mL}$ & $6.3 \%(1)$ & $75.0 \%(12)$ & $18.8 \%(3)$ & \\
CD4 ${ }^{+}$T-cells & & & & \\
$\geq 500$ cells $/ \mathrm{mm}^{3}$ & $22.0 \%(9)$ & $63.4 \%(26)$ & $14.6 \%(6)$ & 0.10 \\
200-499 cells $/ \mathrm{mm}^{3}$ & $12.8 \%(5)$ & $56.4 \%(22)$ & $30.8 \%(12)$ & \\
$<200$ cells $/ \mathrm{mm}^{3}$ & $6.7 \%(1)$ & $86.7 \%(13)$ & $6.7 \%(1)$ & \\
ART & & & & \\
Without PI & $25.0 \%(1)$ & $75.0 \%(3)$ & $0.54(0)$ & 0.54 \\
With PI & $14.1 \%(13)$ & $64.1 \%(59)$ & $21.7 \%(20)$ & \\
\hline
\end{tabular}

ART: antiretroviral therapy.

PI: protease inhibitors. significant association, emphasizing that HLA-E staining in the placentas of the infected and uninfected women was not affected by HIV-1 infection.

\section{Discussion}

HLA-E is expressed in the placental tissues and has immunomodulatory properties. The effect of HIV-1 infection on HLA-E expression, however, has not yet been studied. Therefore, in this study, we evaluated HLA-E expression in the placental tissues of HIV-1-infected and uninfected women.

The expression of HLA nonclassical class I molecules in the placental tissues has been associated with one of the many factors that collaborate with the immunological tolerance during pregnancy, indicating that these molecules may inhibit the cytotoxic activity of uterine NK cells, guaranteeing a successful pregnancy [11]. Our results showed that HLA-E molecules were expressed in the EVT, endothelial, and Hofbauer cells, but not in the syncytiotrophoblast. Menier et al. [12] analyzed the expression of HLA-E in the first trimester placentas of uninfected women and showed that 
HLA-E was expressed in the EVT, endothelial, and Hofbauer cells, but not in the perivillous trophoblast and syncytiotrophoblast.

It has been reported that HIV-1 induces the expression of HLA-E in lymphocytes to inhibit the host's immune system. The expression can be regulated by the HIV-1 p24 $14-22$ peptide, and its interaction is mediated by the CD94/NKG2A inhibitory receptor of NK cells. This interaction results in the inhibition of NK cytotoxic activity, representing an evasion strategy used by HIV-1 [8]. In addition, Nattermann et al. [7] demonstrated that lymphocytes infected with HIV-1 in vitro induced the expression of HLA-E and decreased the cytotoxic activity of NK cells, potentially contributing to the establishment of a chronic infection. At the placenta level, our results show that the presence of HIV-1 infection did not interfere with the expression of HLA-E. Possibly, the control of viral load during pregnancy may have influenced this result once all patients were using ART.

The role of HLA-E and HLA-G in mother-to-child transmission of HIV-1 has been analyzed in women not treated with ART during pregnancy [13-16]. Despite the lack of studies regarding the modulation of HLA-E by ART, the interference of antiretroviral drugs on the modulation of HLA-G expression has been analyzed. Cabello et al. [17] reported an increased number of monocytes expressing HLA-G in patients using antiretroviral drugs when compared to that in untreated patients. Rivero et al. [18] evaluated the expression of HLA-G in different HIV therapeutic regimens and revealed that nucleoside analog reverse-transcriptase inhibitors increased HLA-G expression in circulating $\mathrm{CD} 4^{+}$monocytes and lymphocytes, whereas protease inhibitors did not affect lymphomononuclear cell HLA-G expression. These data are very important because they offer new immunological perspectives on ART, combining the drug pharmacological properties with the modulation of immunomodulatory molecules.

Although there are no studies regarding HLA-E expression in the placental tissue, stratified according to HIV-1 viral load and CD4 ${ }^{+} \mathrm{T}-$ cell count, the evaluation of another immunomodulatory molecule showed that the placental expression of HLA-G1 in HIV-infected women exhibited a significant correlation between maternal viral load and the expression of HLA-G1 [19].

Our results indicated that in the placental tissue of HIV-1infected women with infection under control, the expression of HLA-E in the EVT cells was not modified when compared to that in the placentas of uninfected women. The expression level of HLA-E was not influenced by factors such as viral load, CD4 ${ }^{+}$T-cell count, and use of PI with ART. Although further research studies are needed to understand the influence of HIV-1 on the expression of HLA-E, our results indicate that ART reduces HIV-1 viral load and the peptides that modulate HLA-E expression. Thus, the maintenance of HLA-E expression in HIV-1-infected women may propitiate a pregnancy similar to uninfected women.

\section{Acknowledgements}

We acknowledge the financial support provided by the São
Paulo Research Foundation (FAPESP) (Master's funding in Brazil, FAPESP 11/16885-6).

\section{References}

[1] F. Morandi, V. Pistoia, Interactions between HLA-G and HLA-E in physiological and pathological conditions, Front. Immunol. 5 (2014) 394, http://dx.doi.org/ 10.3389/fimmu.2014.00394.

[2] G. Kochan, D. Escors, K. Breckpot, D. Guerrero-Setas, Role of non-classical MHC class I molecules in cancer immunosuppression, Oncoimmunology 2 (11) (2013) e26491, http://dx.doi.org/10.4161/onci.26491.

[3] M. Dahl, T.V.F. Hviid, Human leucocyte antigen class Ib molecules in pregnancy success and early pregnancy loss, Hum. Reprod. Update 18 (1) (2012) 92-109, http://dx.doi.org/10.1093/humupd/dmr043.

[4] M. Iwaszko, K. Bogunia-Kubik. Clinical Significance of the HLA-E and CD94/ NKG2. Postepy Hig Med Dosw (Online). 2011 Sep 21;65:616-26.

[5] Y.M. Mosaad, Y. Abdel-Dayem, B.S. El-Deek, S.M. El-Sherbini, Association between HLA-E *0101 homozygosity and recurrent miscarriage in Egyptian women, Scand. J. Immunol. 74 (2) (2011 Aug) 205-209, http://dx.doi.org/ 10.1111/j.1365-3083.2011.02559.x.

[6] R.A. Arias, L.D. Muñoz, M.A. Muñoz-Fernández, Transmission of HIV-1 infection between trophoblast placental cells and T-cells take place via an LFA-1mediated cell to cell contact, Virology 307 (2) (2003 Mar 15) 266-277.

[7] J. Nattermann, H.D. Nischalke, V. Hofmeister, B. Kupfer, G. Ahlenstiel G. Feldmann, et al., HIV-1 infection leads to increased HLA-E expression resulting in impaired function of natural killer cells, Antivir. Ther. 10 (1) (2005) 95-107.

[8] P. Tripathi, S. Agrawal, The role of human leukocyte antigen E and G in HIV infection, AIDS 21 (11) (2007 Jul 11) 1395-1404.

[9] X.J. Li, X. Zhang, A. Lin, Y.Y. Ruan, W.H. Yan, Human Leukocyte Antigen-G (HLA-G) expression in cervical cancer lesions is associated with disease progression, Hum. Immunol. 73 (9) (2012 Sep) 946-949, http://dx.doi.org 10.1016/j.humimm.2012.07.041.

[10] SAS Institute Inc, SAS/STAT® User's Guide. Version 9.0, SAS Institute Inc, Cary, NC, 1999.

[11] A.L. Veenstra van Nieuwenhoven, M.J. Heineman, M.M. Faas, The immunology of successful pregnancy, Hum. Reprod. Update 9 (4) (2003 Jul-Aug) 347-357.

[12] C. Menier, B. Saez, V. Horejsi, S. Martinozzi, I. Krawice-Radanne, S. Bruel, et al. Characterization of monoclonal antibodies recognizing HLA-G or HLA-E: new tools to analyze the expression of nonclassical hla class I molecules, Hum. Immunol. 64 (3) (2003 Mar) 315-326.

[13] H.A. Hong, M. Paximadis, G.E. Gray, L. Kuhn, C.T. Tiemessen, Maternal human leukocyte antigen-G (HLA-G) genetic variants associate with in utero motherto-child transmission of HIV-1 in black South Africans, Infect. Genet. Evol. 30 (2015 Mar) 147-158, http://dx.doi.org/10.1016/j.meegid.2014.12.021.

[14] A. Fabris, E. Catamo, L. Segat, M. Morgutti, L.C. Arraes, J.L. de Lima-Filho, et al., Association between HLA-G 3'UTR 14-bp polymorphism and HIV vertical transmission in Brazilian children, AIDS 23 (2) (2009 Jan 14) 177-182, http:// dx.doi.org/10.1097/QAD.0b013e32832027bf.

[15] M. Luo, C. Czarnecki, S. Ramdahin, J. Embree, F.A. Plummer, HLA-G and mother-child perinatal HIV transmission, Hum. Immunol. 74 (4) (2013 Apr) 459-463, http://dx.doi.org/10.1016/j.humimm.2012.11.023.

[16] L. Segat, E. Catamo, A. Fabris, L. Padovan, M. Morgutti, S. Crovella, HLA-G 3 UTR haplotypes and HIV vertical transmission, AIDS 23 (14) (2009 Sep 10) 1916-1918, http://dx.doi.org/10.1097/QAD.0b013e32832f8104.

[17] A. Cabello, A. Rivero, M.J. Garcia, J.M. Lozano, J. Torre-Cisneros, R. González, et al., HAART induces the expression of HLA-G on peripheral monocytes in HIV-1 infected individuals, Hum. Immunol. 64 (11) (2003 Nov) 1045-1049.

[18] A. Rivero, J.M. Lozano, R. González, G. García-Jurado, A. Camacho, J. TorresCisneros, et al., Nucleoside reverse transcriptase inhibitors are able and protease inhibitors unable to induce the tolerogenic molecule HLA-G1 on monocytes from HIV-1 infected patients, Hum. Immunol. 68 (4) (2007 Apr) 303-306.

[19] S. Moodley, R. Bobat, Expression of HLA-G1 at the placental interface of HIV-1 infected pregnant women and vertical transmission of HIV, Placenta 32 (10) (2011 Oct) 778-782, http://dx.doi.org/10.1016/j.placenta.2011.07.012. 